\title{
Exploring the Potential of Short-Baseline Physics at Fermilab
}

\section{Pedro Pasquini*广}

Instituto de Física Gleb Wataghin - UNICAMP, 13083-859, Campinas SP, Brazil

E-mail: pasquinieifi.unicamp.br

\section{O. G. Miranda}

Departamento de Física, Centro de Investigación y de Estudios Avanzados del IPN

Apdo. Postal 14-740 07000 Mexico, DF, Mexico

E-mail: omrefis.cinvestav.mx

\section{Tórtola ${ }^{\S}$}

AHEP Group, Institut de Física Corpuscular - C.S.I.C./Universitat de València, Parc Cientific de Paterna.

C/Catedratico José Beltrán, 2 E-46980 Paterna (València) - SPAIN

E-mail: mariameific.uv.es

\section{J. W. F. Valle}

AHEP Group, Institut de Física Corpuscular - C.S.I.C./Universitat de València, Parc Cientific

de Paterna.

C/Catedratico José Beltrán, 2 E-46980 Paterna (València) - SPAIN

E-mail: valledific.uv.es

\begin{abstract}
We study the capabilities of the short baseline neutrino program at Fermilab to probe the unitarity of the lepton mixing matrix. We find that the Fermilab program can improve the sensitivity to non-unitary parameter $\left|\alpha_{21}\right|$ by about one order of magnitude. Motivated by the future DUNE experiment, we have also analyzed the potential of a liquid Argon near the detector in the LBNF beamline. This will substantially improve the current sensitivity of non-unitarity.
\end{abstract}

The 20th International Workshop on Neutrinos (NuFact2018)

12-18 August 2018

Blacksburg, Virginia

\footnotetext{
* Speaker.

${ }^{\dagger}$ Thanks to G. V. Stenico for providing the SBND GloBES code for the detectors, the FAPESP-CAPES funding grant 2014/05133-1, 2014/19164-6 and 2015/16809-9 and also the partial support from FAEPEX funding grant, No 2391/17.

${ }_{\ddagger}^{\ddagger}$ Thanks the support by CONACyT and SNI (Mexico)

§ Thanks Ramón y Cajal contract (MINECO).

IJFWV and MT thanks the Spanish grants FPA2017-85216-P and SEV-2014-0398 (MINECO), PROMETEOII/2014/084 and GV2016-142 grants from Generalitat Valenciana.
} 


\section{The short-baseline program at Fermilab}

The neutrino oscillation program at Fermilab occupies a central role in neutrino physics experimentation $[1,2]$. Here we aim to study the physics capabilities of the short baseline neutrino program to probe beyond standard model physics associated with the non-unitarity of the lepton mixing matrix. We have also studied the perspectives of the DUNE liquid Argon near the detector to further improve this sensitivity. For a more detailed discussion of the subject (including NSI and sterile neutrino), please refer to the original reference [3]. We studied two configurations:

(1) The Fermilab Short Baseline Neutrino Experiment (SBNE). This experiment consists of three detectors: the Short Baseline Neutrino Detector (SBND), with a baseline of $0.11 \mathrm{~km}$, the $\mu$ BooNE detector, with a baseline of $0.47 \mathrm{~km}$, and the ICARUS detector, whose baseline is $0.6 \mathrm{~km}$. Their size and characteristics are described in the SBNE proposal [4].

(2) The DUNE near detector: The Long-Baseline Neutrino Facility (LBNF) will need a near detector to achieve its main goals. Such detector will receive the highest-intensity neutrino beam in the world sitting at a distance of around 600 meters. The design of such a detector is still under discussion.

For our simulation of SBN, we used the configuration provided by [5]. For the DUNE near detector, we re-scaled the usual DUNE far detector provided by Globes [6]. Since we are insterested in a zero-distance effect, the flux shape and cross section uncertanties are important. In order to take them into consideration we used the following $\chi^{2}$ function

$$
\chi^{2}=\sum_{O=1}^{3} \sum_{i=1}^{N_{\mathrm{bin}}}\left(\frac{N_{i O}^{\exp }-\left(1-a-a_{i O}\right) N_{i O}^{\mathrm{th}}-\left(1-b-b_{i O}\right) N_{i O}^{\mathrm{bg}}}{\sqrt{N_{i O}^{\exp }}}\right)^{2}+\chi_{\mathrm{SYS}}^{2},
$$

where $\chi_{\mathrm{SYS}}^{2}$ contain Gaussian priors for the uncertanties parameters $a, b, a_{i}, b_{i}$ with uncorrelated errors $\sigma . a_{i}$ and $b_{i}$ wrap all the uncertanties in the shape of the detected spectrum, including crosssection. $N_{i O}^{\text {exp }}$ is the total number of events assuming standard physics. $N_{i O}^{\text {th }},\left(N_{i O}^{\mathrm{bg}}\right)$ is the expected number of signal (background) events in non-unitary of experiment $O$.

\section{Sensitivity to New Physics}

Non-Unitarity effects can be described by modifying the (unitary) mixing matrix $U$ by its nonunitary version $U_{N o n-U n i}=\alpha . U$, where $\alpha$ is a triangular matrix $\left(\alpha_{i j}=0\right.$ if $\left.j>i\right)$ that contains the violations of unitarity generated by the existence of heavy neutrino states. At short distances, the neutrino flux is modified by non-oscillatory transitions $v_{\mu} \rightarrow v_{e}$ resulting in an electron neutrino flux of $\phi_{e}^{N o n-U n i} \sim \phi_{e}^{0}+\left|\alpha_{21}\right|^{2} \phi_{\mu}^{0}$, where $\phi_{a}^{0}$ are the flux of neutrinos $a=e, \mu$ in the unitary case.

Hence, short-baseline experiments can constrain $\left|\alpha_{21}\right|$ if fluxes and cross-sections are known to some degree. In Fig. 1 we plotted this sensitivity by assuming overall normalization error of $10 \%$ and shape error of $1 \%$. Notice that the high statistics of LBNF provides one order of magnitude better sensitivity over the SBN. Nevertheless, both improve current limit from NOMAD $\left|\alpha_{21}\right|^{2}<$ $7 \times 10^{-4}$ [7]. The most limiting factor to probe non-unitary in a near detector lies in knowing the shape of the neutrino flux/cross-section with high precision. 


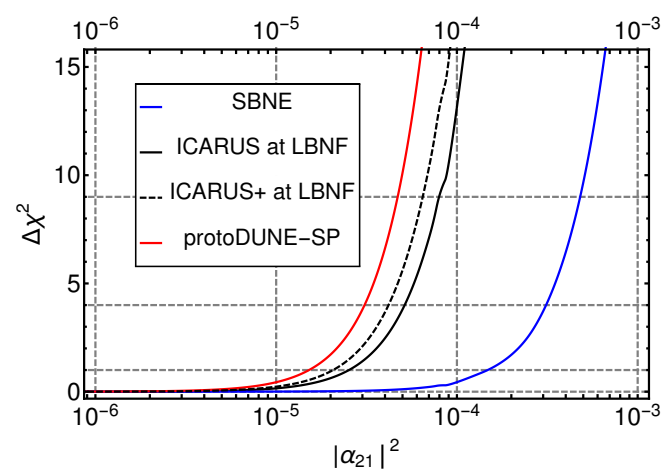

Figure 1: Sensitivity of each configuration assumed: SBN experiment (blue), ICARUS at LBNF (black-solid), ICARUS+ at LBNF (blackdashed) and protoDUNE-SP (red). All of them are assumed to be located at $600 \mathrm{~m}$ from the neutrino source and running for 3.5 years in the neutrino and 3.5 in the anti-neutrino mode.

The DUNE collaboration plan to predict the flux from the muons and hadron-production responsible for the neutrino beam [1], while precise theoretical calculations of the neutrino crosssection is an ongoing debate. In Fig. 2 we present the change in the sensitivity on $\left|\alpha_{21}\right|^{2}$ at $90 \% \mathrm{C}$. L. when varying the detector's Baseline and the shape-spectrum error.

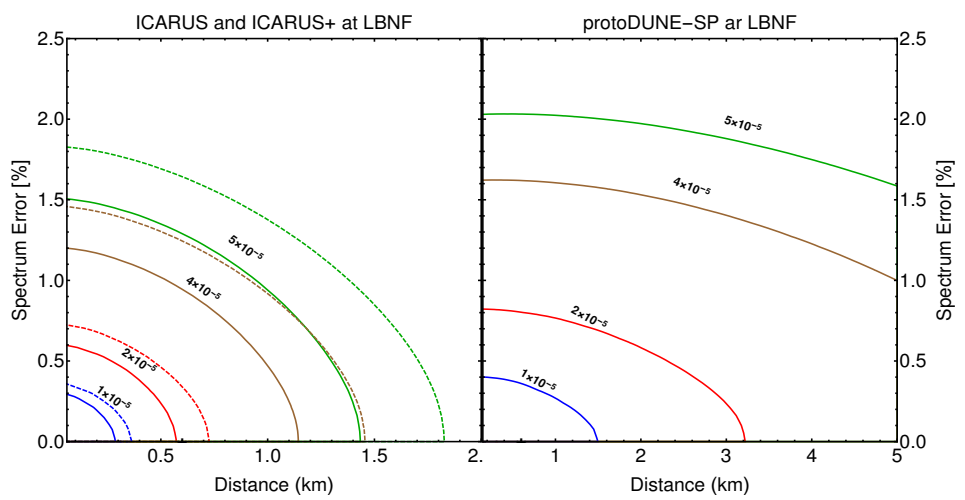

Figure 2: Left: $90 \%$ C.L. sensitivity to $\left|\alpha_{21}\right|$ for ICARUS (solid line) and ICARUS+ (dashed line). Right: $90 \%$ C.L. protoDUNE-SP sensitivity for various combinations of spectrum error. Lines correspond to $\left|\alpha_{21}\right|^{2}<10^{-5}$ (blue), $2 \times 10^{-5}$ (red), $4 \times 10^{-5}$ (brown) and $5 \times$ $10^{-5}$ (green).

\section{Discussion and conclusion}

We looked into the potential capabilities of the SBN program and the near detector of the DUNE experiment to probe non-unitarity. We obtained that it is possible to improve current bounds depending on how well the systematics of the neutrino beam is controlled. If the shape of the spectrum is known at few $\%$, the DUNE near detector could improve the bound on $\left|\alpha_{21}\right|$ by one order of magnitude.

\section{References}

[1] R. Acciarri et al. [DUNE Collaboration], arXiv:1601.02984 [physics.ins-det].

[2] R. Acciarri et al. [DUNE Collaboration], arXiv:1512.06148 [physics.ins-det].

[3] O. G. Miranda, P. Pasquini, M. Tórtola and J. W. F. Valle, Phys. Rev. D 97, no. 9, 095026 (2018).

[4] A. M. Szelc [SBND Collaboration], JINST 11, no. 02, C02018 (2016).

[5] G. V. Stenico, D. V. Forero, and O. L. G. Peres, JHEP 1811, 155 (2018).

[6] P. Huber, J. Kopp, M. Lindner, M. Rolinec, and W. Winter, Comput. Phys. Commun. 177, 432 (2007).

[7] P. Astier et al. [NOMAD Collaboration], Phys. Lett. B 570, 19 (2003). 